\title{
TXNIP overexpression suppresses proliferation and induces apoptosis in SMMC7221 cells through ROS generation and MAPK pathway activation
}

\author{
JIAN LI, ZHONGYI YUE, WANCHENG XIONG, PEISHENG SUN, KUN YOU and JIANGUO WANG
}

\author{
Department of General Surgery, the First Affiliated Hospital, \\ Xinxiang Medical University, Xinxiang, Henan 453100, P.R. China
}

Received September 25, 2016; Accepted March 7, 2017

DOI: $10.3892 / o r .2017 .5577$

\begin{abstract}
Thioredoxin binding protein (thioredoxin-interacting protein, TXNIP), known as vitamin D3 increase protein 1, has been identified as a tumor suppressor in various cancers such as pancreatic, breast, lung and thyroid cancer. However, the role of TXNIP in hepatocellular carcinoma cell proliferation and apoptosis remains unknown. In this study, we first used qRT-PCR, western blotting and immunohistochemistry to compare the expression of TXNIP between hepatocellular carcinoma tissues and tumor-adjacent normal liver tissues. In vitro, we explored the role of TXNIP in hepatocellular carcinoma progression via transfection of the pcDNA-3.1-TXNIP plasmid into SMMC7221 cells. Our results showed that the expression of TXNIP was significantly decreased in hepatocellular carcinoma tissues. Moreover, TXNIP overexpression inhibited hepatocellular carcinoma cell proliferation and induced apoptosis by triggering mitochondrial-mediated ROS generation and activating MAPK pathways. This study provides insight into the molecular mechanisms of TXNIP overexpression in liver cancer cell survival and apoptosis and indicated that TXNIP may be a novel promising agent for liver cancer treatment.
\end{abstract}

\section{Introduction}

Hepatocellular carcinoma (HCC) is one of the most common cancers worldwide and causes approximately 600,000 deaths every year $(1,2)$. Although liver transplantation and surgical resection are the most efficient therapeutic strategies for early stage $\operatorname{HCC}(3,4)$, the majority of patients, who are usually diagnosed at an advanced stage, must rely on

Correspondence to: Dr Jianguo Wang, Department of General Surgery, the First Affiliated Hospital, Xinxiang Medical University, 88 Jiankang Road, Xinxiang, Henan 453100, P.R. China

E-mail: jianguowangjgw@163.com

Key words: thioredoxin-interacting protein, proliferation, apoptosis, SMMC7221 traditional chemotherapies (5). Currently, conventional systemic chemotherapies are still not effective to treat patients with advanced HCC (6). Therefore, novel therapeutic agents with high efficacy are urgently required for the clinical treatment of advanced HCC.

As is known, the abnormal proliferation of cancer cells plays an important role in the development of cancers such as HCC (7). Therefore, apoptosis is considered as a potent therapeutic target for cancer treatment. It is believed that apoptosis can be triggered by diverse pro-apoptosis stimuli converging on mitochondria, which induces mitochondrial dysfunction such as mitochondrial depolarization, cytochrome c release, and caspase enzyme activation $(8,9)$. In addition, mitochondria are the primary source of endogenous reactive oxygen species (ROS) and the targets of ROS in turn (9). Tumor cells with a higher level of ROS can cause redox imbalance, oxidative DNA damage and caspase-dependent or -independent activation of the mitochondria-mediated intrinsic apoptotic pathway (10). Therefore, novel therapeutic agents with high potential in promoting ROS production are urgently required for the clinical treatment of advanced HCC.

Thioredoxin interaction protein (TXNIP) is a $46 \mathrm{kDa}$ protein that was first identified in an HL-60 leukemia cell line treated with $1 \alpha, 25$-dihydroxyvitamin $\mathrm{D}_{3}\left[1,25\left(\mathrm{OH}_{2}\right)\right.$ $\mathrm{D}_{3}$ ] (11). As a negative regulator of thioredoxin (TRX), TXNIP was found to be an inducer of the intracellular level of reactive oxygen species (ROS) (12). A number of studies have confirmed that TXNIP shows strong growth suppressive, metastasis inhibitory and proapoptotic functions $(13,14)$. Subsequently, it has been identified as a tumor suppressor gene in various hematological malignancies and solid tumors, such as breast cancer and thyroid cancer (15-17). Overexpression of TXNIP inhibited tumor growth and caused cell cycle arrest and apoptosis. TXNIP was also shown to play a role as a transcriptional repressor of cyclin A2. TXNIP-deficient cells were shown to upregulate Wnt pathway, reduce p21 and regulate hematopoietic stem cell proliferation and cell quiescence $(18,19)$. However, the potential role of TXNIP in the development of HCC remains unclear.

In this study, we first examined the expression of TXNIP in adjacent non-tumor liver tissue and HCC tissues. We found that the expression of INPP4B directly correlated with TNM 
stage in patients with HCC. Furthermore, we transfected SMMC7221 cells with a pcDNA-3.1-TXNIP plasmid to study the effect and mechanism of TXNIP on the proliferation and apoptosis of HCC cells in vitro. Our clinical and mechanistic data indicated that TXNIP may be a tumor suppressor involved in the progression of HCC. Targeting the TXNIP pathway may be a potential treatment modality for HCC.

\section{Materials and methods}

Patients and tissue samples. From January 2012 to December 2015, 105 HCC patients with liver tumor who underwent surgical resection in Department of General Surgery, the First Affiliated Hospital, Xinxiang Medical University were collected. Thirty matched HCC tissue and the adjacent nontumor liver tissue (ANLT) specimens were obtained from these HCC patients. Normal liver tissues were obtained from 30 patients with giant hemangioma during hepatic resection. Tissue specimens were fixed by formalin immediately upon collection and then paraffin-embedded. The pathological diagnosis for all cases was made by at least two Board Certified pathologists working at the Department of Pathology in the First Affiliated Hospital of Xinxiang Medical University. Informed consent was obtained from each patient and was approved by the Institute Research Ethics Committee at Cancer Center (Xinxiang Medical University).

Immunohistochemistry. The HCC samples were sectioned at $4 \mu \mathrm{m}$. Sections were deparaffinized with xylene and hydrated using graded alcohol; antigen retrieval and blocking were then performed, and slides were incubated with polyclonal rabbit anti-human TXNIP (Bioworld Technology), followed by incubation with secondary antibodies. Detection was performed by 3,3'-diaminobenzidine (DAB) and hematoxylin. The slides were next evaluated by two pathologists in a blinded fashion. All immunohistochemistry staining was independently assessed by two experienced pathologists. The staining intensity was graded from 0 to 2 ( 0 , no staining; 1 , weak; 2 , strong). The staining extent was graded from 0 to 4 based on the percentage of immunoreactive tumor cells $(0,1-5,6-25$, 26-75 and 76-100\%). A score ranging from 0 to 8 was calculated by multiplying the staining extent score with the staining intensity score, resulting in a low (0-4) level or a high (6-8) level for each sample (20).

Cell lines. L02 cells were obtained from the Cancer Research Institute of Xi'an Jiaotong University. HepG2 cells were purchased from the American Type Culture Collection (ATCC, Rockville, MD, USA). SMCC7221 and HCCLM3 cells were obtained from the Cell Bank of Shanghai Institute of Biochemistry and Cell Biology, Chinese Academy of Sciences (Shanghai, China) and cultured in RPMI-1640 (Invitrogen, Carlsbad, CA, USA) medium containing $10 \%$ fetal bovine serum, $100 \mathrm{U} / \mathrm{ml}$ penicillin, and $10 \mu \mathrm{g} / \mathrm{ml}$ streptomycin at $37^{\circ} \mathrm{C}$ in humidified air containing $5 \% \mathrm{CO}_{2}$.

Plasmid constructs and transfection. The Full-length TXNIP coding region was obtained by RT-PCR amplification of normal human liver cDNA and then subcloned into a pcDNA3.1 vector. For transfection, SMMC7221 cells were cultured to $80 \%$ confluence and transfected with $4 \mu \mathrm{g}$ pcDNA3.1-TXNIP plasmid using Lipofectamine 2000 (Invitrogen) according to the manufacturer's recommendations. The pcDNA-3.1-scramble plasmid was used as a negative control. Cells were harvested after $48 \mathrm{~h}$ for subsequent analysis.

Cell proliferation analysis. Cells $\left(1 \times 10^{5}\right.$ cells/well $)$ were seeded in 96-well plates and cultured for 24, 48 and $72 \mathrm{~h}$. After the cell culture medium was removed, cells were washed three times with phosphate-buffered saline (PBS), and then incubated in $100 \mu 1$ freshly DMEM medium containing MTT $(0.2 \mathrm{mg} / \mathrm{ml})$ at $37^{\circ} \mathrm{C}$ for $4 \mathrm{~h}$. The supernatant was removed and $200 \mu \mathrm{l}$ DMSO was added for $30 \mathrm{~min}$ to dissolve the formazan crystals. The absorbance was measured at a wavelength of $570 \mathrm{~nm}$ using a microplate reader (Thermo Fisher Scientific, Inc., Waltham, UK), and the results were used to calculate the viability of the cells.

Apoptosis analysis. Cell apoptosis was analyzed by flow cytometry based on the Annexin V-FITC/propidium iodide (PI) apoptosis kit (Strong Biotech Corp., Taipei, Taiwan) according to the manufacturer's instructions. Briefly, cells were seeded in a 6 -well plate at the density $3 \times 10^{5}$ cells/well and cultured for $24 \mathrm{~h}$, then harvested by trypsinization and centrifuged at $2000 \mathrm{rpm}$ for $5 \mathrm{~min}$, followed by washing twice with ice-cold PBS. After adding $100 \mu 1$ Annexin V binding buffer, $5 \mu \mathrm{l}$ Annexin $\mathrm{V}$ and $5 \mu \mathrm{l}$ PI, the samples were incubated at room temperature for $15 \mathrm{~min}$ in the dark, followed by the addition of Annexin $\mathrm{V}$ binding buffer to bring the total volume to $1 \mathrm{ml}$. The cells were then transferred into a flow cytometry tube and analyzed using a flow cytometer (Cytomics FC 500, Beckman Coulter, Atlanta, CA, USA).

Determination of ROS production. The oxidant sensitive fluorescent probe DCFH-DA was used to determine intracellular ROS generation. Cells were seeded in a 96-well plate at the density $1 \times 10^{4}$ cells/well and cultured for $24 \mathrm{~h}$, then incubated with DCFH-DA for $30 \mathrm{~min}$ at $37^{\circ} \mathrm{C}$ in the dark. After being washed twice with PBS, fluorescence images of the cells were acquired using the InCell 2000 confocal microscope. For the quantitative evaluation of intracellular ROS production efficacy, the fluorescence was measured by the software modules supplied with the InCell 2000.

Measurement of the mitochondrial membrane potential. The mitochondrial membrane potential was measured using JC-1, which was a mitochondria-specific lipophilic cationic fluorescence dye and was capable of selectively entering the mitochondria. Cells were seeded in a 96-well plate at the density $1 \times 10^{4}$ cells/well and cultured for $24 \mathrm{~h}$, then the cell culture media was removed and incubated with $100 \mu \mathrm{l}$ JC-1 stain solution $(200 \mu \mathrm{M})$ for $20 \mathrm{~min}$ at $37^{\circ} \mathrm{C}$ in the dark. After washing twice with PBS, the cells on coverslips were mounted onto slides and examined with the InCell 2000 confocal microscope. Quantitative image analysis was carried out using the software modules supplied with the InCell 2000.

Mitochondrial complex II activity. For mitochondrial complex II activity assay, SMMC7221 cells were cultured in 6 -well plates at the density $1 \times 10^{4}$ cells/well for $24 \mathrm{~h}$. The 

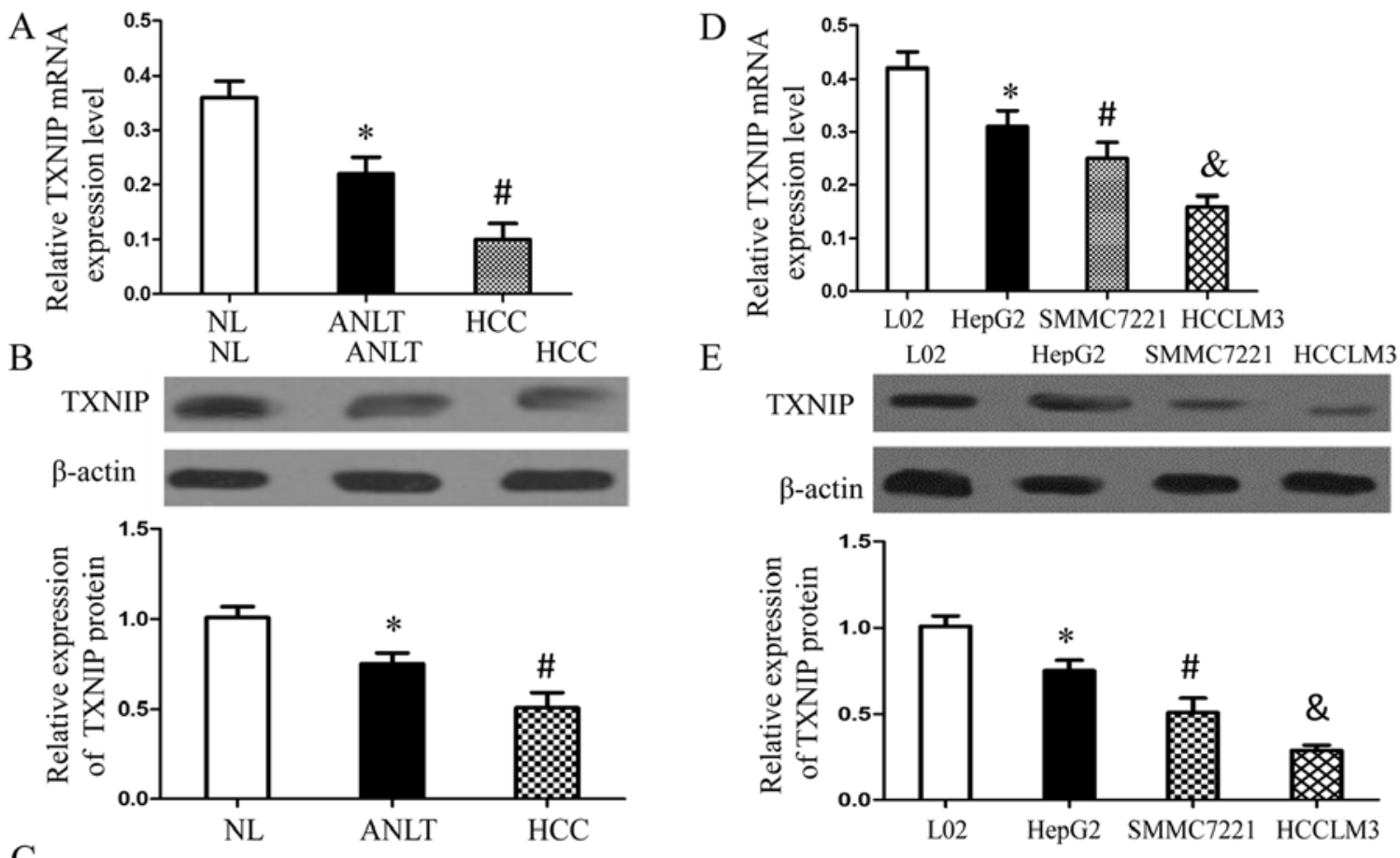

$\mathrm{C}$
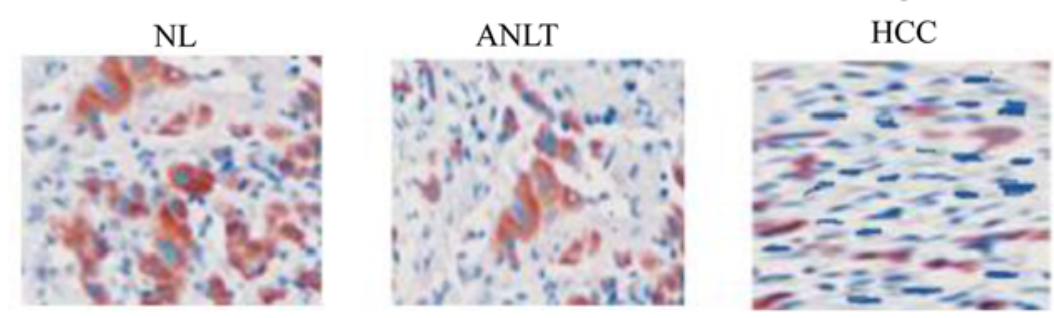

Figure 1. Expression level of TXNIP in HCC tissue and HCC cell lines. (A) The mRNA level of TXNIP was determined by qRT-PCR in HCC tissues (n=30) and in ANLTs $(n=30)$ and in normal liver tissue $(N L)(n=30)$. (B) The protein level of TXNIP was determined by western blotting in HCC tissues, ANLTs and NL. (C) The representative image for TXNIP immunohistochemistry staining in HCC tissues, ANLTs and NL. (D) The mRNA level of TXNIP was determined by qRT-PCR in normal cell lines and HCC cell lines. (E) The protein level of TXNIP was determined by western blotting in normal cell lines and HCC cells lines. ${ }^{*}$ p $<0.05$ vs. NL or L02, ${ }^{\sharp} \mathrm{p}<0.05$ vs. ANLT or HepG2, ${ }^{\circledR} \mathrm{p}<0.05$ vs. SMMC7221.

cells were collected and the activity was detected according to Succinate Dehydrogenase Activity Assay kit (BioVision, Palo Alto, CA, USA). The absorbance was measured at $600 \mathrm{~nm}$.

Quantitative real-time polymerase chain reaction. For $\mathrm{HCC}$ and ANLT tissues, the total RNA was isolated using the RecoverAll ${ }^{\mathrm{TM}}$ Total Nucleic Acid Isolation kit (Ambion, Austin, TX, USA) according to the manufacturer's protocol. Total mRNA of cells was extracted with TRIzol reagent (Invitrogen) according to the manufacturer's instructions. First strand cDNA was generated from $2 \mu \mathrm{g}$ total RNA using RevertAid $^{\mathrm{TM}}$ First Strand cDNA Synthesis kit (Fermentas, Glen Burnie, MD, USA). qRT-PCR was performed in triplicate utilizing the CFX Real-time PCR Detection System (Bio-Rad, Hercules, CA, USA) and Absolute Blue QPCR SYBR Green Mix (Applied Biosystems, Foster City, CA, USA). The cycle number when the fluorescence first reached a preset threshold $(\mathrm{Ct})$ was used to quantify the initial concentration of individual templates for expression of the mRNA of genes of interest. Transcripts of the housekeeping gene GAPDH in the same incubations were used for internal normalization. Primer pairs were as follows: TXINP-F: 5'-TGGCAAAGGAGCAGATT AGTTGCTTAGG-3', and TXNIP-R: 5'-CTGCCACAAGA ACTCTGTGAAATTGGATATTAGA-3'; and GAPDH-F:
5'-CATGCGAACAGCAGTCACTTGCTGATGTAT-3', and GAPDH-R: 5'-ATTTTAGGACCCTGCGTCGGCCGTCTG-3'.

Western blot analysis. Cells were homogenized in ice-cold radio immunoprecipitation assay lysis buffer (Santa Cruz Biotechnology, Santa Cruz, CA, USA) for $30 \mathrm{~min}$ and centrifuged at $12,000 \mathrm{x} \mathrm{g}$ at $4^{\circ} \mathrm{C}$ for $10 \mathrm{~min}$, and the supernatants were collected. Protein concentrations were determined using a protein assay dye reagent (Bio-Rad). Equal amounts (usually $50 \mu \mathrm{g}$ ) were loaded onto a 10\% SDS-PAGE gel and separated electrophoretically. Then, the proteins were transferred to a PVDF membrane (Millipore, Boston, MA, USA). After blocking with 5\% non-fat dry milk in PBS with $0.1 \%$ Tween-20 (PBS-T) for $2 \mathrm{~h}$ at room temperature, the PVDF membrane was incubated overnight at $4^{\circ} \mathrm{C}$ with primary antibodies, including anti-SDHA (1:1500, Santa Cruz Biotechnology), anti-Bax (1:1000, Cell Signaling Technology, Danvers, MA, USA), anti-Bcl-2 (1:1000, Cell Signaling Technology), anti-cleaved caspase-9 (1:1000, Cell Signaling Technology), anti-cleaved caspase-3 (1:1000, Cell Signaling Technology), anti-p38 (1:1000, Cell Signaling Technology), anti-p-p38 (1:1000, Cell Signaling Technology), anti-ERK (1:1000, Cell Signaling), anti-p-ERK (1:1000, Cell Signaling Technology), anti-JNK (1:1500, Cell Signaling Technology), and anti-p-JNK 


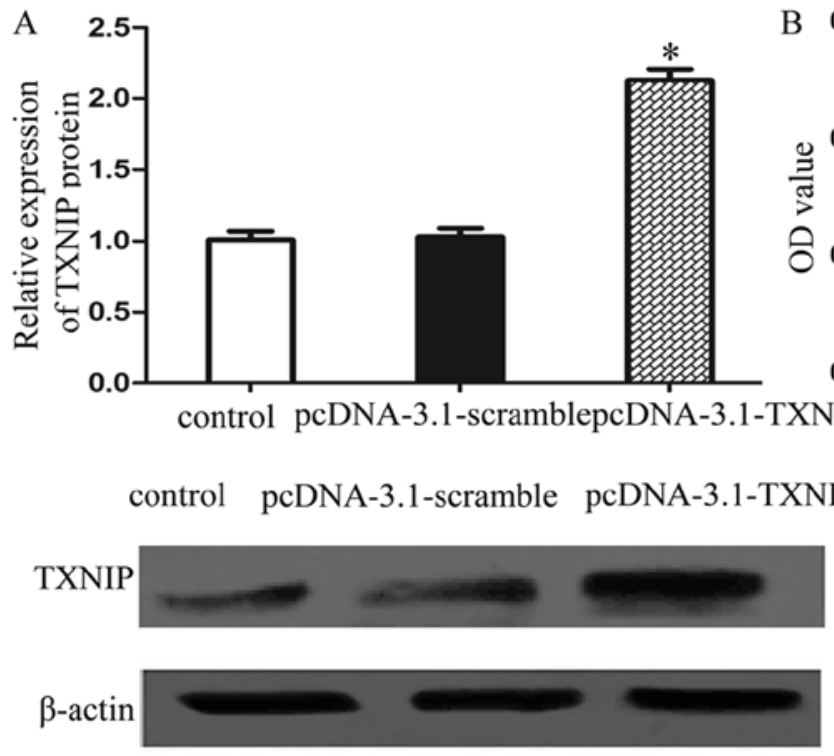

$\mathrm{C}$

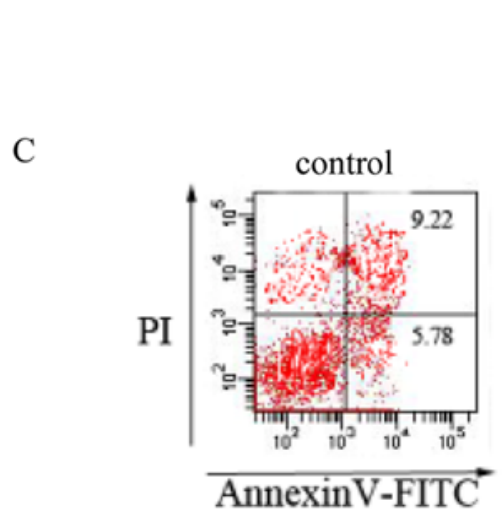

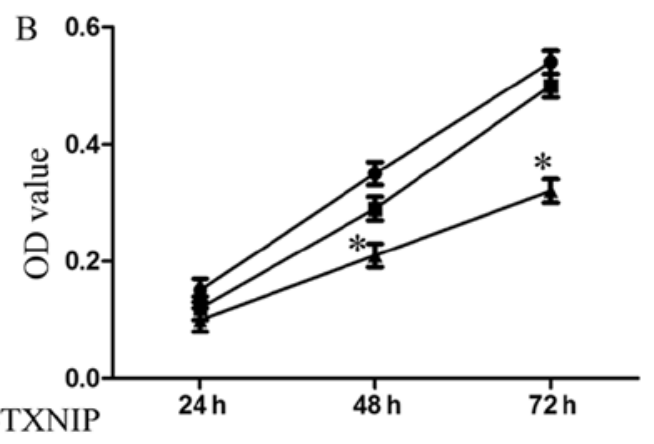

- control

$\rightarrow$ pcDNA-3.1-scramble

$\mp$ pcDNA-3.1-TXNIP

$\mathrm{D}$

cleaved Caspase-9

cleaved Caspase- 3
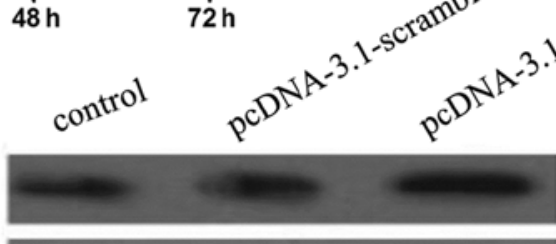

$\operatorname{Bax}$

Bcl-2

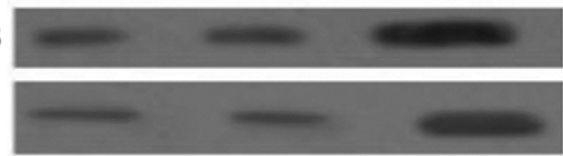

$\beta$-actin
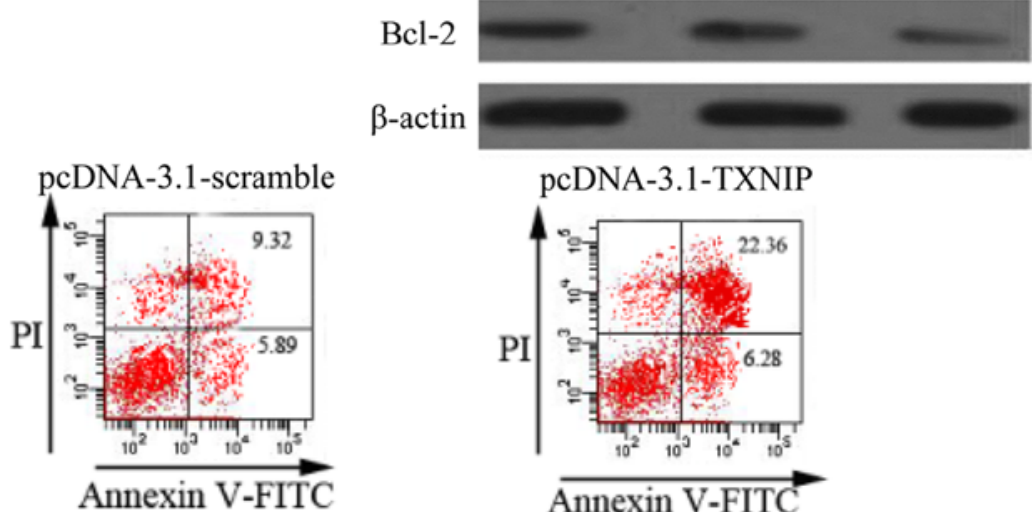

pcDNA-3.1-TXNIP

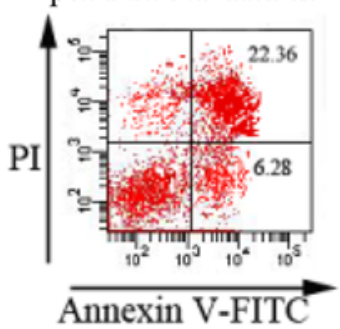

Figure 2. Overexpression of TXNIP inhibits HCC cell proliferation and induces apoptosis. (A) The protein level of TXNIP was measured by western blotting. (B) The proliferation of SMMC7221 cells was measured by MTT analysis. (C) SMMC7221 cells apoptosis were measured using Annexin V and propidium iodine (PI) staining. (D) The protein levels of apoptosis-related proteins were measured by western blotting. "p $<0.05$ vs. control.

(1:1000, Cell Signaling Technology), respectively, followed by incubation with horseradish peroxidase (HRP)-conjugated goat anti-rabbit IgG antibodies (1:2000, Sigma, St. Louis, MO, USA) for $1 \mathrm{~h}$ at room temperature. Protein detection was performed based on an enhanced chemiluminescence (ECL) method and photographed using a BioSpectrum Gel Imaging System (HR410, UVP, Upland, CA, USA). Detection of $\beta$-actin on the same membrane was used as a loading control.

Statistical analysis. Data are presented as the mean \pm SD. Differences between two groups were analyzed by Student's t-test, while differences between multiple groups were analyzed by ANOVA. P-values $<0.05$ were considered to indicate a statistically significant difference.

\section{Results}

TXNIP expression is reduced in human hepatocellular carcinoma. To determine whether TXNIP expression was changed in human hepatocellular carcinoma, we first examined its mRNA expression and protein expression in HCC tissues and the adjacent non-tumor liver tissues (ANLT) isolated from the same patient as well as in normal liver tissues (NL) obtained from patients with giant hemangioma by qRT-PCR and western blotting, respectively. There were $30 \mathrm{HCC}$ patients randomly selected for the study. At the same time, immunohistochemistry was also performed to analyze the expression of TXNIP in HCC tissues, ANLT and NL. Noteworthy, when compared with the mRNA level and protein level of TXNIP in the ANLT tissues, both were significantly downregulated in HCC tissues (Fig. 1A and B). The results of immunohistochemistry showed that none or few cells showing positively-stained cytoplasm were detected in HCC tissues and diffuse strong brown in ANLT (Fig. 1C).

We further examined the differences of TXNIP expression between three HCC cell lines and a normal liver cell line (L02 cells) by qRT-PCR (Fig. 1D) and western blotting (Fig. 1E). Of note, TXNIP expression in cancer cells was negatively correlated with cell metastatic potential, since HCCLM3 cells possessed the highest capability for metastasis, while HepG2 cells had the least capacity for metastasis. Combined with the above results, we concluded that TXNIP expression was reduced in the human hepatocellular carcinoma.

Correlation of TXNIP expression with clinicopathological characteristics of HCC. Subsequently, the association of 


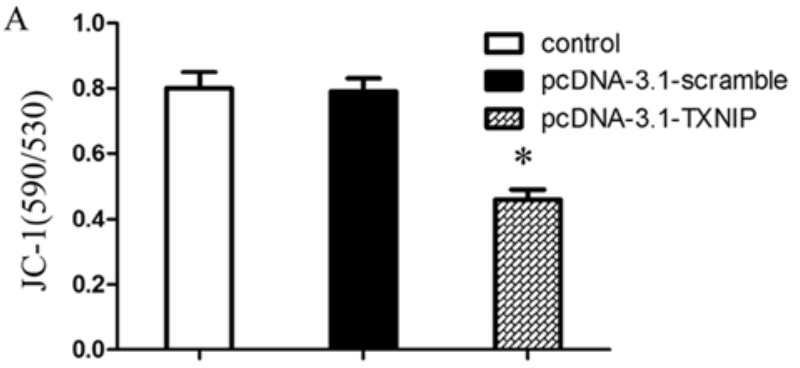

$\mathrm{C}$ control pcDNA-3.1-scramble pcDNA-3.1-TXNIP

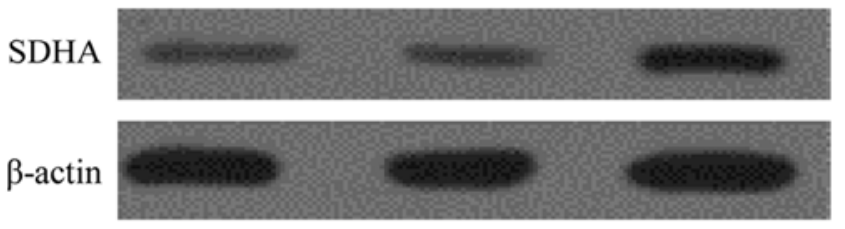

D control
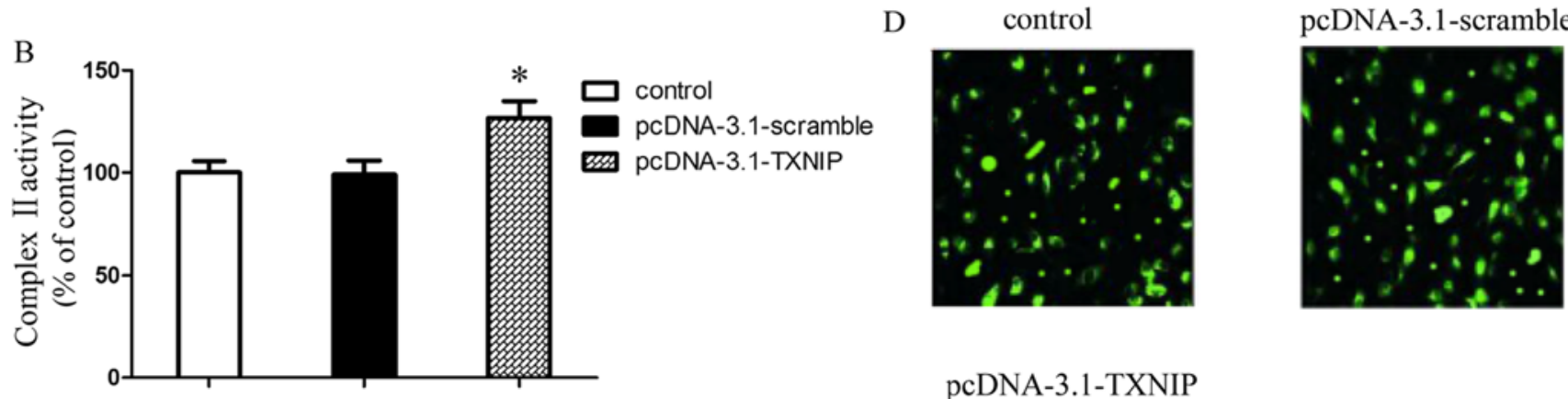

pcDNA-3.1-TXNIP
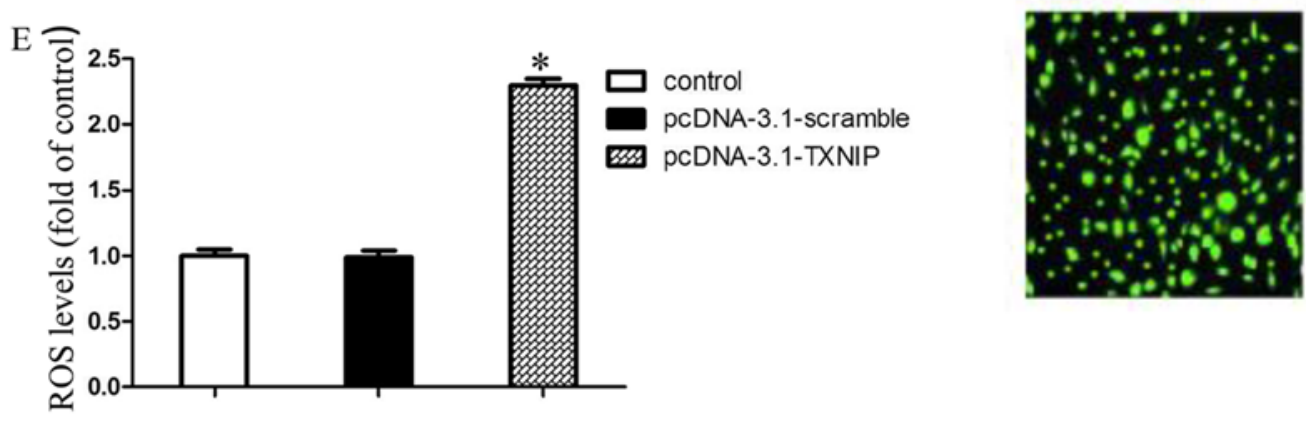

Figure 3. Overexpression of TXNIP increases ROS generation and mitochondrial dysfunction. (A) The mitochondrial membrane potential of SMMC7221 cells was measured using the mitochondrial dye JC-1. (B) The complex II activity of SMMC7221 cells was confirmed by measuring the absorbance of 2, 6-dichlorophenol-indophenol (DCPIP) at $600 \mathrm{~nm}$ according to Succinate Dehydrogenase Activity Assay kit. (C) The protein level of SDHA was measure by western blotting. The ROS production was measured by DCF-DA staining (D) and calculated (E). " p $<0.05$ vs. control.

TXNIP expression with the clinicopathological features of HCC was analyzed. Overall, 105 HCC patients were stratified into the low and high expression groups according to the results of immunohistochemistry. The correlations of TXNIP expression with clinicopathological characteristics were analyzed. It was found that the TXNIP expression was related with tumor nodule number, vascular invasion and TNM (Table I). These results together revealed that decreased TXNIP expression possibly participated in HCC progression.

TXNIP overexpression significantly inhibits SMMC7221 cell proliferation and induces apoptosis. To functionally characterize the role of TXNIP in HCC cell proliferation and apoptosis, MTT assay and flow cytometry were performed. SMMC7221 cells were transfected with pcDNA-3.1-TXNIP plasmid and the TXNIP expression was confirmed by western blot analysis (Fig. 2A). The results of proliferation analysis and apoptosis showed that enforced TXNIP expression inhibited SMMC7221 cells proliferation and induced apoptosis (Fig. 2B and C). Furthermore, TXNIP overexpression increased the expression of cleaved caspase- 9 and -3 , as well as increased the expression of pro-apoptotic protein Bax and suppressed the anti-apoptotic protein Bcl-2 (Fig. 2D). Collectively, these data suggested that TXNIP overexpression induced growth arrest and mitochondrial-related apoptosis in SMMC7221 cells.

TXNIP overexpression increases ROS generation via impaired mitochondrial function. Mitochondrial dysfunction is closely related to the mitochondrial-related apoptosis. In this study, JC-1 staining was performed to detect the mitochondrial membrane potential in SMMC7221 cells. Fig. 3A shows that the mitochondrial membrane potential of SMMC7221 was decreased after transfection with pcDNA-3.1-TXNIP plasmid, which indicated that TXNIP overexpression induced mitochondrial dysfunction. Moreover, TXNIP overexpression significantly increased the mitochondrial complex II activity of SMMC7221 and promoted the expression of SDHA, a subunit of complex II which is a major site for ROS generation (Fig. 3B and C). To determine whether ROS generation was involved in TXNIP overexpression-induced apoptosis, we measured the levels of ROS in SMMC7221-treated cells using DCF-DA reagent (Fig. 3D). Our results revealed that TXNIP overexpression could increase the ROS generation (Fig. 3E). Combined with the above results, we concluded that TXNIP overexpression could increase the ROS generation via impaired mitochondrial function. 
A control
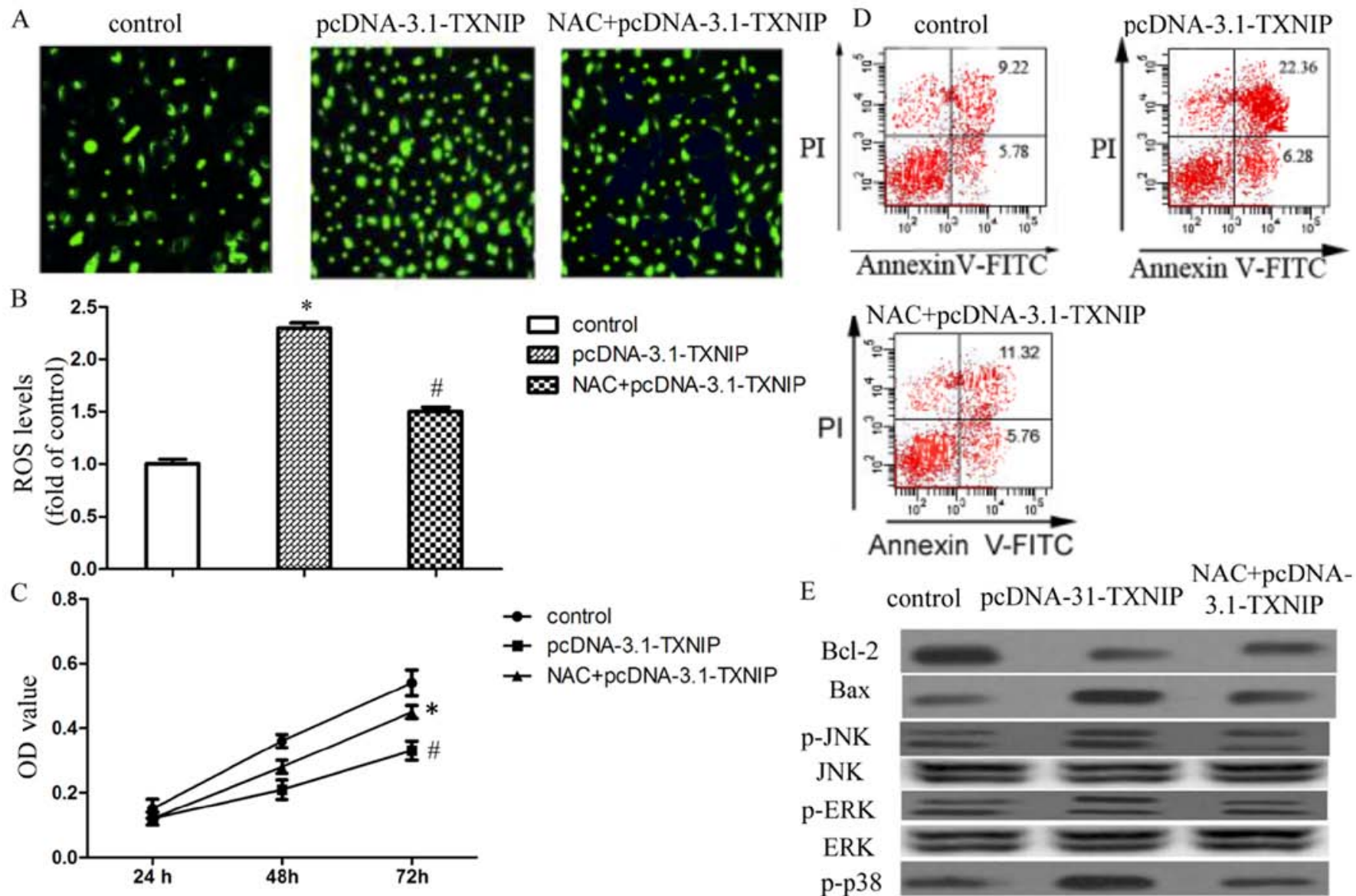

$\mathrm{E}$
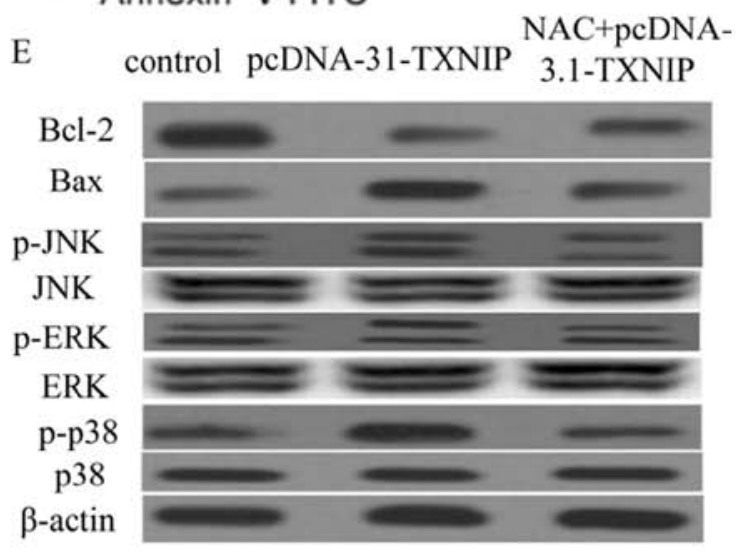

Figure 4. Inhibition of ROS production attenuated TXNIP overexpression-induced apoptosis and MAPK pathway activation in SMMC7221 cells. The ROS production was measured by DCFDA staining (A) and calculated (B). (C) The proliferation of SMMC7221 cells was measured by MTT analysis. (D) SMMC7221 cell apoptosis was measured using Annexin V and propidium iodine (PI) staining. (E) The protein levels of apoptosis-related proteins and MAPKs pathway related proteins were measured by western blotting. ${ }^{*} \mathrm{p}<0.05$ vs. control. ${ }^{*} \mathrm{p}<0.05$ vs. pcDNA-3.1-TXNIP.

ROS generation is involved in TXNIP overexpression-induced SMMC7221 cell growth arrest and apoptosis. In order to assess the roles of ROS generation in TXNIP overexpressioninduced SMMC7221 cell growth arrest and apoptosis, we treated SMMC7221 cells with ROS scavenger NAC before transfection with the pcDNA-3.1-TXNIP plasmid. Our results revealed that NAC markedly attenuated TXNIP-induced ROS generation in SMCC7221 cells (Fig. 4A and B). NAC also alleviated the inhibition effects of TXNIP overexpression on SMMC7221 proliferation, as well as cancer cell apoptosis (Fig. 4C and D). We also examined the effects of NAC on TXNIP overexpression-induced variation of apoptosis-related proteins. Our results revealed that NAC obviously attenuated TXNIP overexpression-induced downregulation of Bcl-2 and upregulation of Bax (Fig. 4E). The results suggested that ROS generation may be involved in TXNIP overexpression-induced growth arrest and apoptosis.

TXNIP overexpression activates MAPK pathways in a ROSdependent manner. We next sought to identify the signal transduction pathways involved in proliferation inhibition in response to TXNIP overexpression. We monitored the expression levels and activation status of MAPKs, which are also the major oxidative stress-sensitive signal transducing pathways and serve as upstream signals for the initiation of apoptosis. The results showed that TXNIP overexpression significantly increased the levels of phosphorylated JNK, ERK and p38 without affecting the expression of total proteins. Interestingly, the ROS scavenger NAC significantly attenuated the TXNIP overexpression-induced activation of JNK, ERK and p38 MAPK (Fig. 4E). Our data suggested that TXNIP overexpression could activate the JNK, ERK and p38 MAPK via ROS.

\section{Discussion}

TXNIP was initially identified as a thioredoxin-binding protein that inhibits thioredoxin (TRX), thereby contributing to redox homeostasis (21). Since TRX also promotes tumor progression by angiogenesis induction (22) and apoptosis inhibition $(23,24)$, TXNIP, as its inhibitor, has great potential as a tumor suppressor gene. Several studies have shown that the expression of TXNIP was significantly reduced in different types of aggressive cancers such as acute myeloid leukemia (AML), breast cancer and bladder cancer, which emphasized its relevance in tumor prevention (25-29). In this study, we found that TXNIP expression was decreased significantly 
Table I. The correlations of TXNIP with clinicopathological features of $\mathrm{HCC}$.

\begin{tabular}{|c|c|c|c|c|}
\hline \multirow[b]{2}{*}{$\begin{array}{l}\text { Clinicopathological } \\
\text { variable }\end{array}$} & \multirow[b]{2}{*}{$\mathrm{n}$} & \multicolumn{2}{|c|}{ TXNIP } & \multirow[b]{2}{*}{ P-value } \\
\hline & & $\begin{array}{c}\text { Low } \\
\text { expression }\end{array}$ & $\begin{array}{c}\text { High } \\
\text { expression }\end{array}$ & \\
\hline Gender & & & & 0.150 \\
\hline Female & 67 & 38 & 29 & \\
\hline Male & 38 & 16 & 22 & \\
\hline Age & & & & 0.296 \\
\hline$<60$ & 46 & 21 & 25 & \\
\hline$\geq 60$ & 59 & 33 & 26 & \\
\hline AFP & & & & 0.393 \\
\hline$<20 \mathrm{ng} / \mathrm{ml}$ & 91 & 45 & 46 & \\
\hline$\geq 20 \mathrm{ng} / \mathrm{ml}$ & 14 & 9 & 5 & \\
\hline HBsAg & & & & 0.872 \\
\hline Negative & 85 & 44 & 41 & \\
\hline Positive & 20 & 10 & 10 & \\
\hline Tumor nodule number & & & & 0.003 \\
\hline Solitary & 31 & 9 & 22 & \\
\hline Multiple (>2) & 74 & 45 & 29 & \\
\hline Vascular invasion & & & & 0.029 \\
\hline Absence & 93 & 34 & 59 & \\
\hline Presence & 12 & 10 & 2 & \\
\hline TNM & & & & 0.039 \\
\hline I & 55 & 23 & 32 & \\
\hline II-III & 50 & 31 & 19 & \\
\hline
\end{tabular}

in most $\mathrm{HCC}$ tissues, suggesting a potential involvement of TXNIP in the development of HCC. It has been reported that TNXIP is an inhibitor of TRX, which could suppress tumor cell apoptosis. Hence, we transfected pcDNA-3.1-TXNIP plasmid into SMMC7221 cells with the purpose of exploring the effect of TXNIP overexpression on HCC cell proliferation and apoptosis.

Our results showed that TXNIP overexpression significantly inhibited SMCC7221 cell proliferation and induced apoptosis (Fig. 2B and C). It has been confirmed that apoptosis could be induced through an intrinsic or extrinsic pathway (30-32), and activation of the caspase cascade was also involved in apoptosis (33). Only when caspase is cleaved can the activation of caspase occur. Furthermore, activation of caspase-9, an initiator caspase, is required for the activation of executioner caspase (i.e., caspase-3) to induce apoptosis (34). In our study, TXNIP overexpression obviously activated caspase- 9 and -3 , which led to the induction of apoptosis. Although it is still unknown whether an intrinsic or extrinsic pathway was the key pathway inducing apoptosis in SMCC7221 cells transfected with pcDNA-3.1-TXNIP plasmid, TXNIP overexpression-induced apoptosis involved the intrinsic pathway that was associated with mitochondrial dysfunction. Mitochondria-dependent apoptosis is regulated by proteins of the Bcl-2 family. Our results showed that TXNIP over- expression promoted the expression of a proapoptotic protein (Bax) and inhibits the expression of an antiapoptotic protein (Bcl-2) (Fig. 2D). In addition, SMMC7221 cells transfected with pcDNA-3.1-TXNIP plasmid exhibited a lower mitochondrial membrane potential (Fig. 3A); combined with the above results, we concluded that TXNIP overexpression could induce apoptosis in HCC cells through mitochondrial dysfunction.

Mitochondria are not only vital organelles for producing ATP and intermediates for eukaryotic cancer cells, they are also key sources of ROS generation (35-37). Mitochondrial complex II, known as succinate dehydrogenase (SDH), is the only complex that participates in the Krebs cycle as well as the electron transport chain (38). Ralph et al (39) reported that the succinate dehydrogenase (SDH)/complex II system could act as a key redox regulator of ROS production via an electron driving mechanism. In our study, TXNIP overexpression significantly increased the activity of complex II and promoted the expression of SDHA, as well as increasing the production of intracellular ROS. It is well known that the intrinsic apoptotic pathway is especially susceptible to ROS (40). In the present study, the inhibition of ROS production by the antioxidant NAC significantly alleviated TXNIP overexpression-mediated growth arrest and apoptosis (Fig. 4C and D), suggesting that TXNIP overexpression-induced apoptosis in SMMC7221 cells was closely associated with the production of ROS, and may act as upstream signaling molecules to initiate mitochondriamediated cell apoptosis.

MAPKs are the major oxidative stress-sensitive signal transducing pathways and are also a family of serine/threonine kinases that regulate a variety of cellular events such as proliferation and apoptosis (41), at the same time serving as upstream signals for the initiation of apoptosis (42). Further, ROS functions as second messengers in diverse signaling pathways (43). Our results showed that TXNIP overexpression significantly increased the levels of phosphorylated JNK, ERK and p38 MAPK without affecting the expression of total proteins (Fig. 4E), indicating that these MAPK pathways were activated in the process of TXNIP overexpression-induced apoptosis in SMMC7221 cells. Further, our study also showed that pre-treatment with NAC prevented the phosphorylation of JNK, ERK and p38 MAPK. Therefore, we concluded that JNK, ERK and p38 MAPK were activated in response to TXNIP overexpression-induced ROS generation, which are frequently associated with the induction of apoptosis.

In summary, we demonstrated that TXNIP overexpression induced apoptotic cell death in SMMC7221 cells through intrinsic pathways via triggering mitochondrial-mediated ROS generation and activating MAPK pathways. This study provided insight into the molecular mechanisms of TXNIP overexpression-induced apoptosis in liver cancer cells and presented that TXNIP may serve as a potential therapeutic target for HCC.

\section{References}

1. Bao H, Liu P, Jiang K, Zhang X, Xie L, Wang Z and Gong P: Huaier polysaccharide induces apoptosis in hepatocellular carcinoma cells through p38 MAPK. Oncol Lett 12: 1058-1066, 2016.

2. Torre LA, Bray F, Siegel RL, Ferlay J, Lortet-Tieulent J and Jemal A: Global cancer statistics, 2012. CA Cancer J Clin 65: 87-108, 2015. 
3. Signoriello S, Annunziata A, Lama N, Signoriello G, Chiodini P, De Sio I, Daniele B, Di Costanzo GG, Calise F, Olivieri G, et al: Survival after locoregional treatments for hepatocellular carcinoma: A cohort study in real-world patients. Sci World J 2012: 564706, 2012

4. Chan SL and Yeo W: Targeted therapy of hepatocellular carcinoma: Present and future. J Gastroenterol Hepatol 27: $862-872,2012$

5. Lu SC: Where are we in the chemoprevention of hepatocellular carcinoma? Hepatology 51: 734-736, 2010.

6. Wang X, Wang N, Cheung F, Lao L, Li C and Feng Y: Chinese medicines for prevention and treatment of human hepatocellular carcinoma: Current progress on pharmacological actions and mechanisms. J Integr Med 13: 142-164, 2015.

7. Li H, Miao Q, Xu CW, Huang JH, Zhou YF and Wu MJ: OTX1 contributes to hepatocellular carcinoma progression by regulation of ERK/MAPK pathway. J Korean Med Sci 31: 1215-1223, 2016.

8. Sepand MR, Ghahremani MH, Razavi-Azarkhiavi K, Aghsami M, Rajabi J, Keshavarz-Bahaghighat $\mathrm{H}$ and Soodi M: Ellagic acid confers protection against gentamicin-induced oxidative damage, mitochondrial dysfunction and apoptosisrelated nephrotoxicity. J Pharm Pharmacol 68: 1222-1232, 2016.

9. Zeng CC, Lai SH, Yao JH, Zhang C, Yin H, Li W, Han BJ and Liu YJ: The induction of apoptosis in HepG-2 cells by ruthenium(II) complexes through an intrinsic ROS-mediated mitochondrial dysfunction pathway. Eur J Med Chem 122 118-126, 2016

10. Diao QX, Zhang JZ, Zhao T, Xue F, Gao F, Ma SM and Wang Y: Vitamin E promotes breast cancer cell proliferation by reducing ROS production and p53 expression. Eur Rev Med Pharmacol Sci 20: 2710-2717, 2016.

11. Zhu HJ, Wang DG, Yan J and Xu J: Up-regulation of microRNA135a protects against myocardial ischemia/reperfusion injury by decreasing TXNIP expression in diabetic mice. Am J Transl Res 7: 2661-2671, 2015.

12. Hong K, Xu G, Grayson TB and Shalev A: Cytokines regulate $\beta$-cell thioredoxin-interacting protein (TXNIP) via distinct mechanisms and pathways. J Biol Chem 291: 8428-8439, 2016.

13. Hong SY and Hagen T: 2-Deoxyglucose induces the expression of thioredoxin interacting protein (TXNIP) by increasing O-GlcNAcylation - Implications for targeting the Warburg effect in cancer cells. Biochem Biophys Res Commun 465: 838-844, 2015.

14. Chen D, Dang BL, Huang JZ, Chen M, Wu D, Xu ML, Li R and Yan GR: MiR-373 drives the epithelial-to-mesenchymal transition and metastasis via the miR-373-TXNIP-HIF1 $\alpha$-TWIST signaling axis in breast cancer. Oncotarget 6: 32701-32712, 2015

15. Baldan F, Mio C, Lavarone E, Di Loreto C, Puglisi F, Damante G and Puppin C: Epigenetic bivalent marking is permissive to the synergy of HDAC and PARP inhibitors on TXNIP expression in breast cancer cells. Oncol Rep 33: 2199-2206, 2015.

16. Nie W, Huang W, Zhang W, Xu J, Song W, Wang Y, Zhu A, Luo J, Huang G, Wang Y, et al: TXNIP interaction with the Her-1/2 pathway contributes to overall survival in breast cancer. Oncotarget 6: 3003-3012, 2015.

17. Morrison JA, Pike LA, Sams SB, Sharma V, Zhou Q, Severson JJ, Tan AC, Wood WM and Haugen BR: Thioredoxin interacting protein (TXNIP) is a novel tumor suppressor in thyroid cancer. Mol Cancer 13: 62, 2014

18. Filios SR, Xu G, Chen J, Hong K, Jing G and Shalev A MicroRNA-200 is induced by thioredoxin-interacting protein and regulates Zeb1 protein signaling and beta cell apoptosis. J Biol Chem 289: 36275-36283, 2014

19. Ho B, Huang G and Golubovskaya VM: Focal adhesion kinase regulates expression of thioredoxin-interacting protein (TXNIP) in cancer cells. Anticancer Agents Med Chem 14: 3-8, 2014.

20. Liu Y, Zhang JB, Qin Y, Wang W, Wei L, Teng Y, Guo L, Zhang B, Lin Z, Liu J, et al: PROX1 promotes hepatocellular carcinoma metastasis by way of up-regulating hypoxia-inducible factor 10 expression and protein stability. Hepatology 58: 692-705, 2013.

21. Nishiyama A, Matsui M, Iwata S, Hirota K, Masutani H, Nakamura H, Takagi Y, Sono H, Gon Y and Yodoi J: Identification of thioredoxin-binding protein-2/vitamin $\mathrm{D}(3)$ up-regulated protein 1 as a negative regulator of thioredoxin function and expression. J Biol Chem 274: 21645-21650, 1999.

22. Welsh SJ, Bellamy WT, Briehl MM and Powis G: The redox protein thioredoxin-1 (Trx-1) increases hypoxia-inducible factor 1alpha protein expression: Trx-1 overexpression results in increased vascular endothelial growth factor production and enhanced tumor angiogenesis. Cancer Res 62: 5089-5095, 2002.
23. Saitoh M, Nishitoh H, Fujii M, Takeda K, Tobiume K, Sawada Y, Kawabata M, Miyazono K and Ichijo H: Mammalian thioredoxin is a direct inhibitor of apoptosis signal-regulating kinase (ASK) 1. EMBO J 17: 2596-2606, 1998.

24. Liu Y and Min W: Thioredoxin promotes ASK1 ubiquitination and degradation to inhibit ASK1-mediated apoptosis in a redox activity-independent manner. Circ Res 90: 1259-1266, 2002.

25. Zhou J, Bi C, Cheong LL, Mahara S, Liu SC, Tay KG, Koh TL, Yu Q and Chng WJ: The histone methyltransferase inhibitor, DZNep, up-regulates TXNIP, increases ROS production, and targets leukemia cells in AML. Blood 118: 2830-2839, 2011.

26. Hirotsu C, Tufik S, Bergamaschi CT, Tenorio NM, Araujo P and Andersen ML: Sleep pattern in an experimental model of chronic kidney disease. Am J Physiol Renal Physiol 299: F1379-F1388, 2010.

27. Sheth SS, Bodnar JS, Ghazalpour A, Thipphavong CK, Tsutsumi S, Tward AD, Demant P, Kodama T, Aburatani $\mathrm{H}$ and Lusis AJ: Hepatocellular carcinoma in Txnip-deficient mice. Oncogene 25: 3528-3536, 2006

28. Butler LM, Zhou X, Xu WS, Scher HI, Rifkind RA, Marks PA and Richon VM: The histone deacetylase inhibitor SAHA arrests cancer cell growth, up-regulates thioredoxin-binding protein-2, and down-regulates thioredoxin. Proc Natl Acad Sci USA 99: $11700-11705,2002$.

29. Nishizawa K, Nishiyama H, Matsui Y, Kobayashi T, Saito R, Kotani H, Masutani H, Oishi S, Toda Y, Fujii N, et al: Thioredoxin-interacting protein suppresses bladder carcinogenesis. Carcinogenesis 32: 1459-1466, 2011.

30. Fox JL and MacFarlane M: Targeting cell death signalling in cancer: Minimising 'Collateral damage'. Br J Cancer 115: 5-11, 2016.

31. Matthews GM, Newbold A and Johnstone RW: Intrinsic and extrinsic apoptotic pathway signaling as determinants of histone deacetylase inhibitor antitumor activity. Adv Cancer Res 116: 165-197, 2012.

32. Wajant H: The Fas signaling pathway: More than a paradigm. Science 296: 1635-1636, 2002.

33. Shimizu H, Banno Y, Sumi N, Naganawa T, Kitajima Y and Nozawa Y: Activation of p38 mitogen-activated protein kinase and caspases in UVB-induced apoptosis of human keratinocyte HaCaT cells. J Invest Dermatol 112: 769-774, 1999.

34. Wu YJ, Wong BS, Yea SH, Lu CI and Weng SH: Sinularin induces apoptosis through mitochondria dysfunction and inactivation of the $\mathrm{pI} 3 \mathrm{~K} / \mathrm{Akt} / \mathrm{mTOR}$ pathway in gastric carcinoma cells. Mar Drugs 14: 14, 2016.

35. Zong WX, Rabinowitz JD and White E: Mitochondria and Cancer. Mol Cell 61: 667-676, 2016.

36. Weinberg SE and Chandel NS: Targeting mitochondria metabolism for cancer therapy. Nat Chem Biol 11: 9-15, 2015.

37. Byun HO, Kim HY, Lim JJ, Seo YH and Yoon G: Mitochondrial dysfunction by complex II inhibition delays overall cell cycle progression via reactive oxygen species production. J Cell Biochem 104: 1747-1759, 2008

38. Wang L, Zhang X, Cui G, Chan JY, Wang L, Li C, Shan L, Xu C, Zhang Q, Wang Y, et al: A novel agent exerts antitumor activity in breast cancer cells by targeting mitochondrial complex II. Oncotarget 7: 32054-32064, 2016.

39. Ralph SJ, Moreno-Sánchez R, Neuzil J and Rodríguez-Enríquez S: Inhibitors of succinate: Quinone reductase/Complex II regulate production of mitochondrial reactive oxygen species and protect normal cells from ischemic damage but induce specific cancer cell death. Pharm Res 28: 2695-2730, 2011.

40. Zhang C, Jia X, Bao J, Chen S, Wang K, Zhang Y, Li P, Wan JB, $\mathrm{Su} \mathrm{H}$, Wang Y, et al: Polyphyllin VII induces apoptosis in HepG2 cells through ROS-mediated mitochondrial dysfunction and MAPK pathways. BMC Complement Altern Med 16: 58, 2016.

41. Takeda K, Matsuzawa A, Nishitoh H and Ichijo H: Roles of MAPKKK ASK1 in stress-induced cell death. Cell Struct Funct 28: 23-29, 2003.

42. Zebelo SA and Maffei ME: Role of early signalling events in plant-insect interactions. J Exp Bot 66: 435-448, 2015.

43. Avisetti DR, Babu KS and Kalivendi SV: Activation of p38/JNK pathway is responsible for embelin induced apoptosis in lung cancer cells: Transitional role of reactive oxygen species. PLoS One 9: e87050, 2014. 